\title{
Design of Vector Control System for Brushless DC motor Based on Hall Sensor
}

\author{
Lihui Sun ${ }^{1,}$, , Jun $\mathrm{Yu}^{2, \mathrm{~b}}$ \\ ${ }^{1}$ School of Information and Control Engineering, Jilin Institute of Chemical Technology, Jilin \\ 132022, China \\ ${ }^{2}$ Dean`s Office, Jilin Institute of Chemical Technology, Jilin 132022, China \\ asunlihui2000@126.com, b83770573@qq.com
}

\begin{abstract}
Most of audio players on the market use the hardware filter Abstract. As one kind of special motor, Brushless DC motor has characteristics of high efficiency, simple control, wide application range and others. The traditional brushless DC motor adopts square wave control, which cannot meet the noise requirements in some application fields. In this paper, a vector control system of brushless motor based on Hall sensor is introduced, and the system structure is introduced from the hardware and software of the system. The experimental results show that the vector control system introduced in this paper can greatly improve the efficiency and reduce the operation noise.
\end{abstract}

Keywords: Hall Sensor, motor, Vector Control.

\section{Introduction}

As one kind of special motor, Brushless DC motor has characteristics of high efficiency, simple control, wide application range and others. Under the requirements of energy saving and emission reduction today, brushless DC motor is gradually replacing the original low efficiency AC asynchronous motor, which is widely used in the fields of fan, water pump, household electrical appliance and automobile air conditioning etc. The traditional brushless DC motor is controlled by square wave, which has large torque ripple and large phase change noise and cannot meet the requirement of noise in some application fields. The use of vector control technology can greatly improve the efficiency and reduce the operation noise.

\section{The Overall Design Scheme of the System}

Brushless DC motor vector control system is composed of power supply, controller, motor, speed regulator, PC and other components. The power supply provides the necessary power for the controller, and the controller, motor and speed regulator jointly fulfill the application requirements of the control system. After the system is powered on, self-check is made to ensure that the controller can work normally. The controller processes according to the signal issued by the speed regulator, for example, after the starting signal is obtained, the motor starts to run. During the operation of the motor, the motor state information, such as the speed can be sent to the PC by the serial port.

\section{The Hardware Design of the System}

The hardware system of the brushless DC motor vector control system is composed of motor, drive circuit, control circuit, detection circuit, power circuit and so on. The hardware system structure is shown in Figure1 the driving circuit adopts three-phase bridge type two-level voltage inverter structure three-phase Inverter Bridge, which has 6 power switch devices, and the switch devices select High-performance Power MOSFET. The system control circuit uses the ST Company STM32F103 as the control chip. On the one hand, the control circuit will collect voltage, current and position signal to complete the vector control algorithm. On the other hand, the motor start stop control is completed according to the potentiometer signal. The detection circuit mainly includes phase current, bus current and Holzer position sensor signal detection, and the current and position information obtained are used to complete the vector control algorithm. The power circuit converts the external 
output DC power into three independent power sources $+14 \mathrm{~V},+5 \mathrm{~V}$ and $+3.3 \mathrm{~V}$, and provides stable power supply to the circuit board.

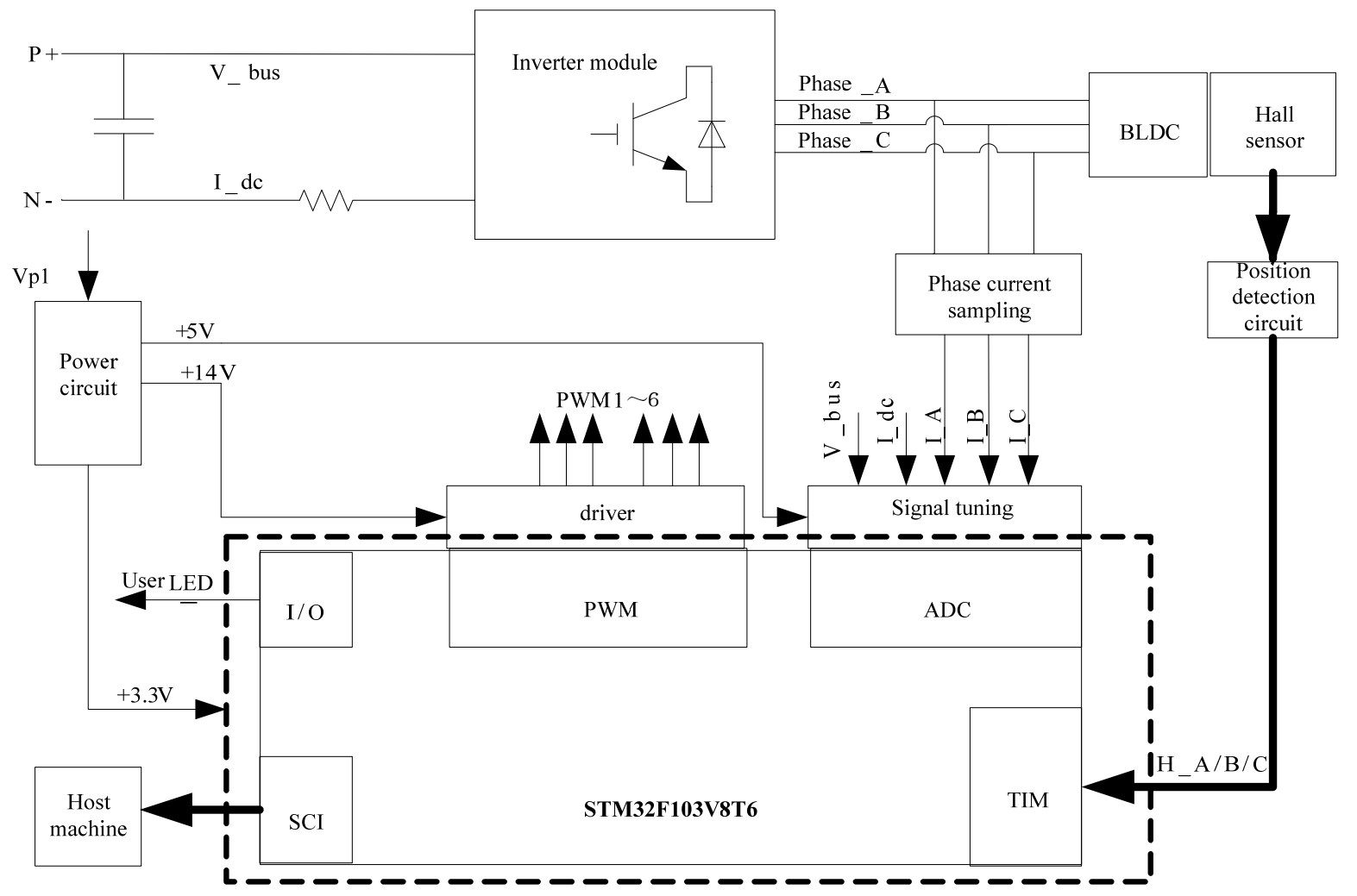

Figure 1. Hardware design of the system

\subsection{Power Supply Circuit Design}

The power supply circuit is composed of a $+14 \mathrm{~V}$ voltage conversion circuit, a $+5 \mathrm{~V}$ voltage conversion circuit and a $+3.3 \mathrm{~V}$ voltage conversion circuit. The input voltage is input to the LM317 converter chip by the current limiting resistor RP1, and the $+14 \mathrm{~V}$ voltage is obtained to provide power for the driver chip IR2136. $+14 \mathrm{~V}$ the $+14 \mathrm{~V}$ power supply is then passed through the 7805 -conversion chip to get $+5 \mathrm{~V}$, which provides power for the peripheral circuit of the system. The $+5 \mathrm{~V}$ power supply is then passed through the AS117 converter chip to get the $+3.3 \mathrm{~V}$ power supply for the microcontroller, and the schematic diagram of the power supply circuit is shown in Figure 2.

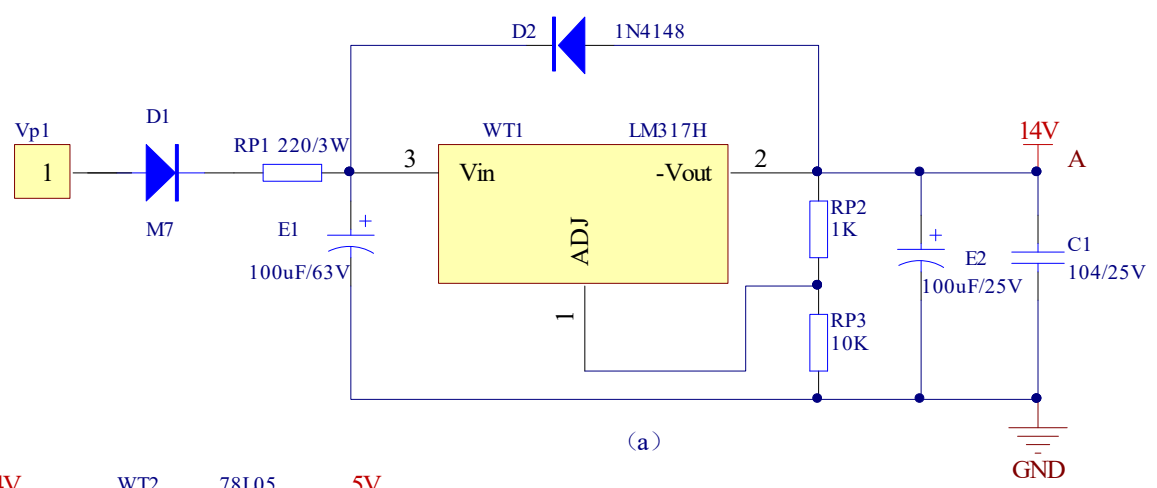

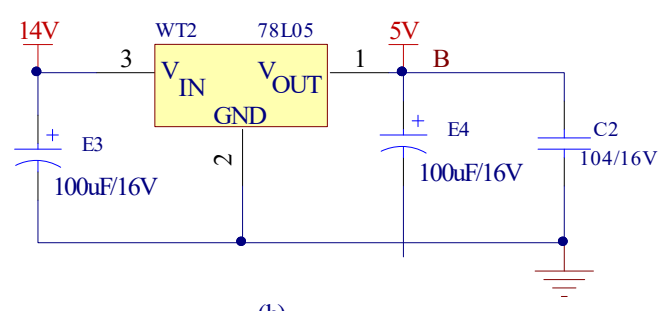

(b)

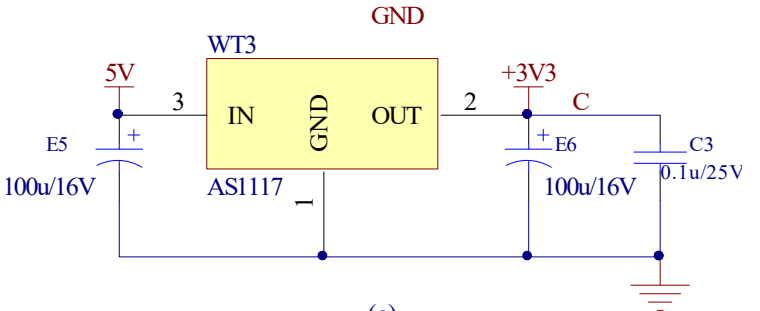

(c)

Figure 2. Power supply circuit design 


\subsection{Design of Motor Driving Circuit}

The driving circuit is as shown in Figure 3. In the motor control power drive circuit, the threephase inverter bridge circuit has 6 power switching devices. If each power switch device is driven by an independent circuit, then it requires 6 driving circuits, which increases the complexity of the circuit and reduces the reliability. The chip IR2136 is a special gate drive circuit of the switch tube, which can drive a power switch device with a bus voltage up to $600 \mathrm{~V}$. It has 3 independent high voltage and low voltage output channels. The internal of it uses bootstrap technology, which requires only one DC power supply to output the driving pulse of the 6-circuit power switch device, which simplifies the design of the driving circuit. The chip IR2136 has a built-in dead zone circuit, as well as over current protection and undervoltage protection circuits. The control logic input of the IR2136 is compatible with the CMOS and TTL electric levels, and the noise filter is input at the same time which has a good noise suppression capability.

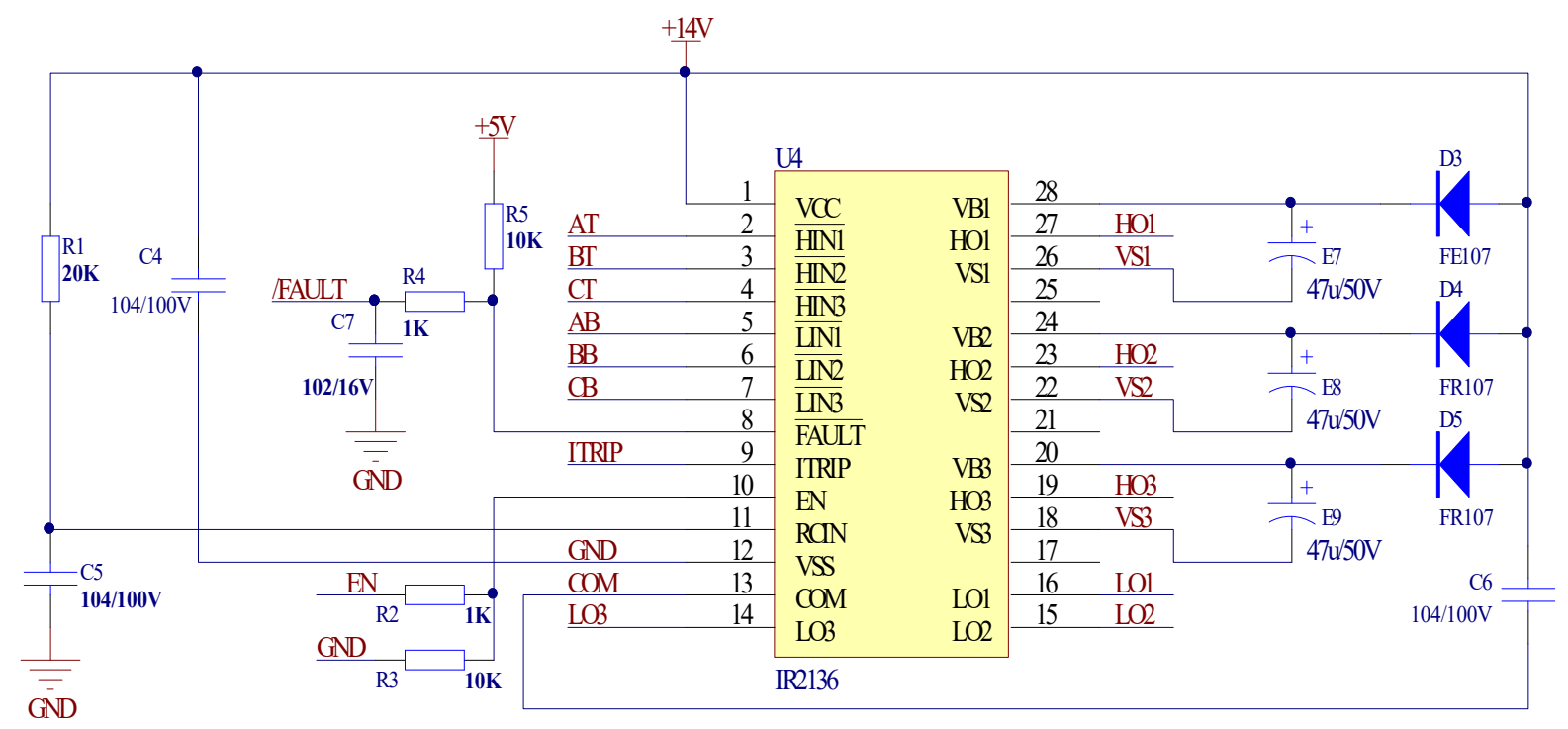

Figure 3. Driving circuit design

\subsection{Design of Current Detection Circuit}

Design of phase current detection circuit

Figure 4 is the schematic diagram of the $A B$ phase current detection circuit. The front part of the circuit is the Hall current sensor detection circuit, and the latter part is the first order active low pass filter circuit. R11 and C13 constitute first order filter circuit, TS924 is voltage follower, which keeps input and output resistance matching. 

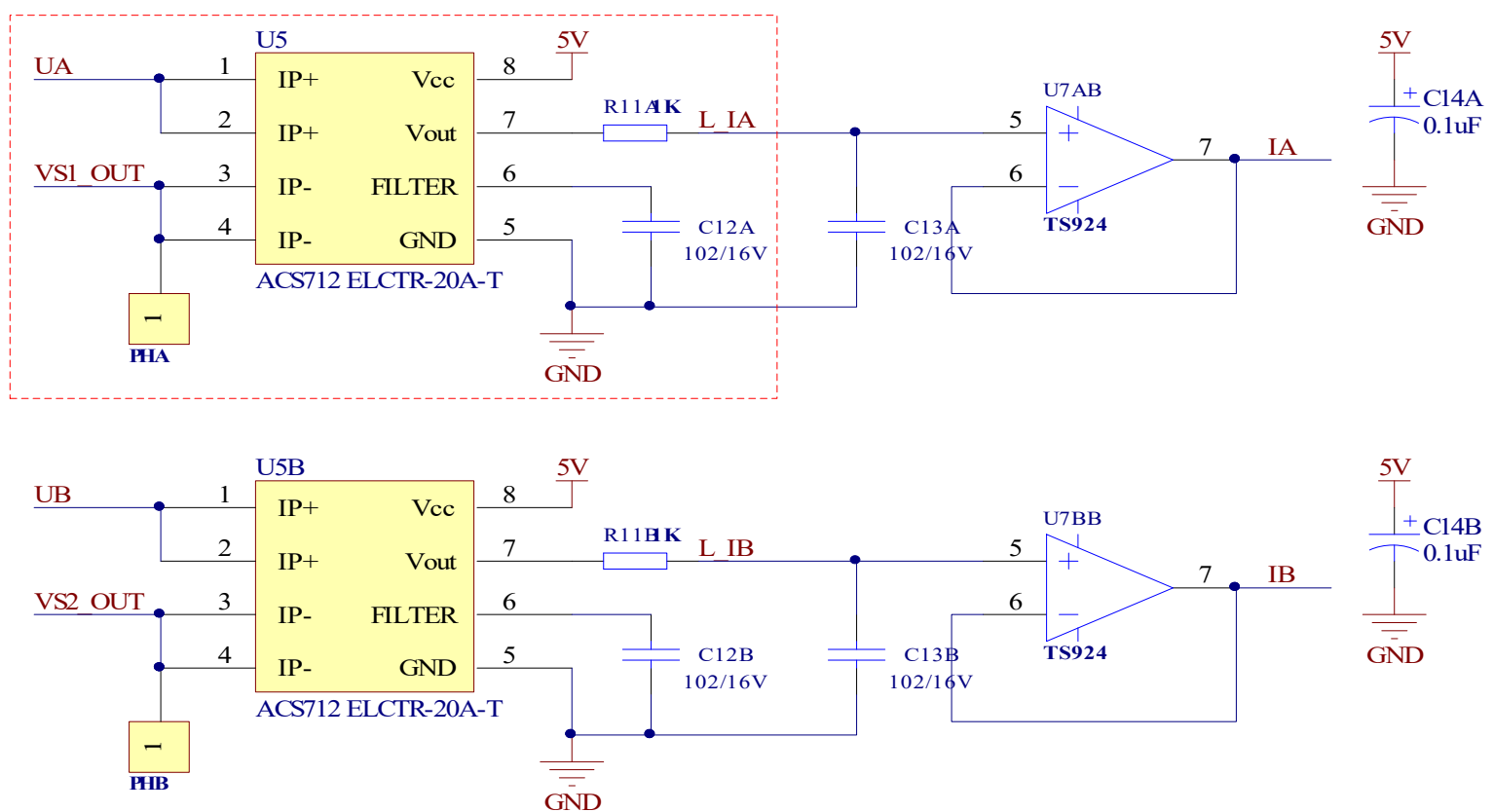

Figure 4. AB phase current detection circuit design

Bus current detection circuit

The bus current collection circuit is shown in Figure 5. When the motor starts or stops, the bus current will increase. In order to ensure the safe and reliable operation of the motor, the bus current collection circuit is added to monitor the current value of the motor in any time. The bus current acquisition circuit converts the current signal to the voltage signal by using the detection resistor in series on the DC bus, and then it is sent to the ADC input channel of the microcontroller after being processed by the amplifier. The current in the inverter circuit is grounded through the sampling resistor RS1 and produces a voltage drop on the RS1. The voltage drop is sampled for the A/D of the MCU after being amplified by an operational amplifier, therefore indirectly obtaining the corresponding current value.

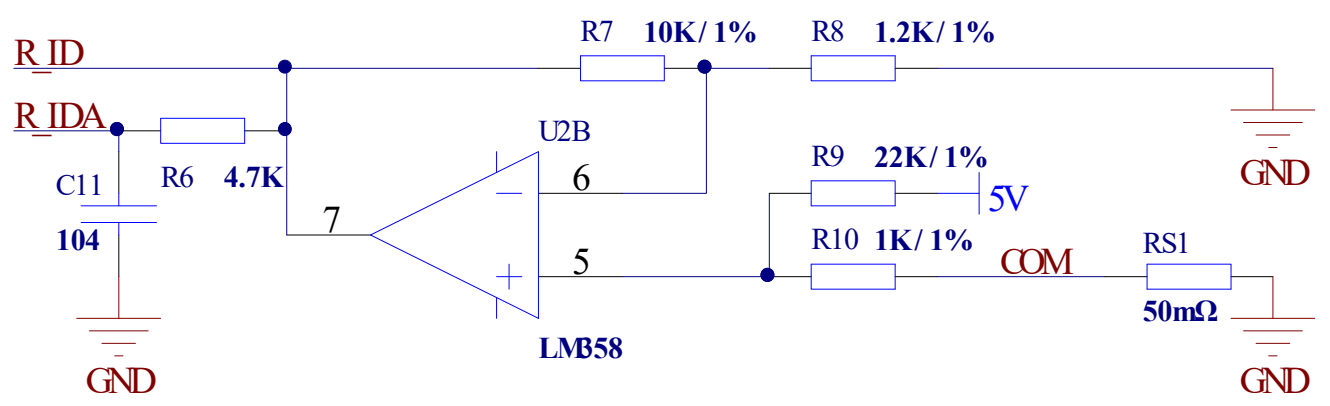

Figure 5. Design of bus current detection circuit

As the detection resistor RS1 is in series in the power circuit, in order to avoid the detection resistor producing a large extra loss, detection resistance need to be very small, where the selection of resistance for $50 \mathrm{~m} \Omega$ as a detection resistor. Calculate the amplification times as $A v=1+R 7 / R 8=9.33$, the bus current after being filtered through R6, C11, we get the average bus current.

According to the virtual short virtual break principle:

$$
\begin{gathered}
\frac{V_{\mathrm{cc}}-V_{5}}{R_{9}}=\frac{V_{5}-V_{\mathrm{com}}}{R_{10}} \\
V_{5}=\frac{V_{\mathrm{cc}}+R_{9} \times V_{\mathrm{com}}}{R_{9}+R_{10}} \\
V_{\text {out }}=\left(1+\frac{R_{7}}{R_{8}}\right) V_{5}
\end{gathered}
$$


We can get from formula (1), (2) and (3):

$$
V_{\text {out }}=\left(1+\frac{R_{7}}{R_{8}}\right)\left(\frac{V_{c c}+R_{9} \times V_{c o m}}{R_{9}+R_{10}}\right)
$$

The bias output voltage can be obtained from it

$$
V_{\text {ref }}=\left(1+\frac{R_{7}}{R_{8}}\right) \times V_{5}
$$

When there is no current, the bias voltage $\operatorname{Vref}=5.0 / 23 \mathrm{~K} \times(1+10 / 1.2)=2.02 \mathrm{~V}$

$$
\Delta V_{\text {out }}=\left(1+\frac{R_{7}}{R_{8}}\right)\left(\frac{R_{9} \times V_{\text {com }}}{R_{9}+R_{10}}\right)
$$

Using resistance of $50 \mathrm{~m} \Omega$, when the bus current is $1 \mathrm{~A}, \Delta$ Vout $\approx 0.5 \mathrm{~V}$.

\subsection{Power Supply Voltage Detection Circuit}

Fig.6 is the schematic diagram of the voltage detection circuit. The bus voltage is directly divided by voltage divider RP5 and RP6, and after voltage division and being filtered by C13, it is directly sent to the AD conversion module of the microcontroller.

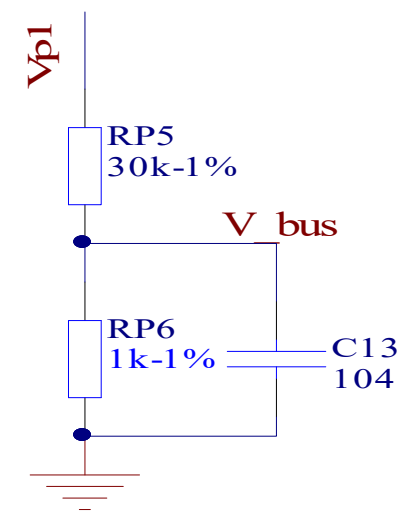

Figure 6. Voltage detection circuit

RP5 and RP6 are resistors with a precision of 1\%, and resistance value of 30k and $1 \mathrm{k}$ are chosen respectively. When the supply voltage is $42 \mathrm{~V} \sim 56 \mathrm{~V}$, the input voltage to the AD module of the microcontroller is limited to $0 \sim 3.3 \mathrm{~V}$.

From this we can get the AD sample under voltage threshold value:

$$
V_{\text {LOW }}=42 \times \frac{R P 6}{R P 5+R P 6}=1.35 \mathrm{~V}
$$

AD sample overvoltage threshold value:

$$
V_{H I G}=56 \times \frac{R P 6}{R P 5+R P 6}=1.81 \mathrm{~V}
$$

According to the above calculation, when the sampling value of the supply voltage is less than $1.35 \mathrm{~V}$, it is judged to be under voltage. When the sampling value of the supply voltage is greater than $1.81 \mathrm{~V}$, it is judged to be overvoltage.

\subsection{Design of Position Detection Circuit}

The internal of common used brushless DC Motor Internal adopts the switch type Hall sensor as a position detection element, the principle is: when the magnetic sensitive surface has a magnetic field, the output is low electric level, no magnetic field when output to high level. The output circuit of the switch type Hall sensor is the collector open circuit, so the pull resistor must be connected. The circuit principle is shown in Figure 7. U1, V1 and W1 are the output signals of Hall sensor, and U, V and W signals are supplied to the control chip. 


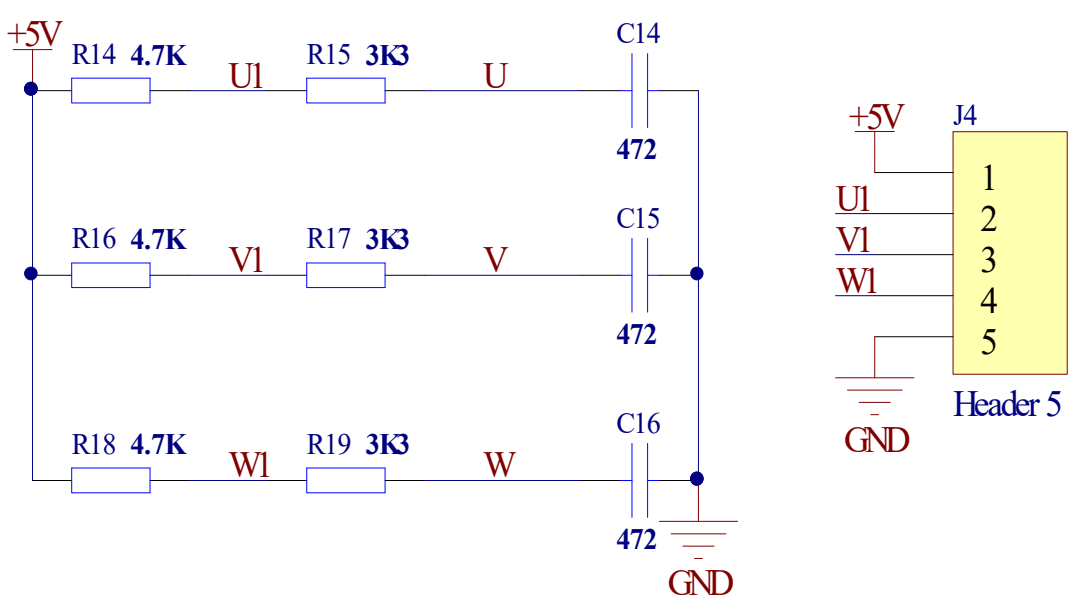

Figure 7. Position detection circuit design

Vector control system is a multithread control system which integrates analog data collection, control volume adjustment and fault protection. It adopts modular design method of real-time control program, fault handling, System state machine, etc. as the foreground program, all levels of interrupt processing as the background program. The software design consists of three parts, the main program, the Interrupt program (ADC interruption, Timer4 interruption and Timer2 interruption), and the control program of the host computer. The main programming design implements the application and the fault control related function, including the control module initialization, the fault processing as well as the program circular execution state mechanism. The interrupt program design realizes the related functions of motor control, including position signal, phase current signal sampling and processing, and execution current and speed closed-loop control etc. The PC control program completes the data interaction between controller and $\mathrm{PC}$, and realizes the tasks of on-line parameter adjustment, data storage and so on.

\section{Software Design of the System}

The software design of the vector control system consists of three parts: the main program, interrupt program and PC control program that start to run after the system is powered on. The main program design implements functions related to application and fault control, including the control of modules initialization, fault handling as we as state mechanism of program cyclic execution. The interrupt program is designed to realize the related functions of motor control, including position signal, phase current signal sampling and processing, and execution current and speed closed-loop control etc. The PC control program completes the data interaction between controller and PC, and realizes the tasks of on-line parameter adjustment, data storage and so on.

The main program design mainly includes system initialization configuration and working state transformation. After the system is powered on, the initial configuration of the variables and the controller external devices is initialized. The system completes the initialization configuration and enters the state machine processing cycle. In the state machine cycle, the system is first carried out to self-check and check the quality of the switch tube. If the switch tube is good, the program continues to execute, otherwise the switch tube fault is prompted by the indicator lamp and waiting for trouble clearance. Then, the software timing task is executed, and the state transformation is queried periodically. The system can display the state at that time through the indicator light.

\section{Conclusion}

In this paper, a DC brushless motor vector control system based on Hall sensor is designed from aspects of hardware and software. The hardware design mainly includes power driving circuit, inverter circuit, voltage and current sampling circuit, power supply circuit, motor phase signal output port and position signal input port, etc. The software design mainly includes main program module 
and interrupt program module. The main program module includes the system initialization module and the motor control state machine module, the Interrupt program module includes Hall detection module, rotor position and speed calculation module, SVPWM space voltage vector modulation module, current control module, PI speed /current regulator module, etc.

\section{Acknowledgments}

This work was financially supported by General project of Jilin Institute of Chemical Engineering 2018066.

\section{References}

[1]. Chen Jifeng, Zhang Xiaofeng, Wu Xiaokang et al, A new strategy of commutation torque ripple suppression for brushless DC motor based on unified method [J]. Journal of Electro technical Technology, 2011, 26 (4): 51 - 56.

[2]. Yang Tao, Li Quanfeng et al, Control method of variable load operation of sensorless brushless DC motor [J]. Journal of Zhejiang University, 2012, 46 (5): 879 - 884.

[3]. Wang Yingfa, Study on commutation torque ripple suppression and position sensorless control of brushless DC motor [D]. Tianjin: Tianjin University, 2012.6. 